\title{
Boundedness for a Class of Singular Integral Operators on Both Classical and Product Hardy Spaces
}

\author{
Chaoqiang Tan \\ Department of Mathematics, Shantou University, Shantou 515063, China \\ Correspondence should be addressed to Chaoqiang Tan; cqtan@stu.edu.cn \\ Received 10 September 2013; Accepted 2 January 2014; Published 27 February 2014 \\ Academic Editor: Henryk Hudzik
}

Copyright ( 2014 Chaoqiang Tan. This is an open access article distributed under the Creative Commons Attribution License, which permits unrestricted use, distribution, and reproduction in any medium, provided the original work is properly cited.

\begin{abstract}
We found that the classical Calderón-Zygmund singular integral operators are bounded on both the classical Hardy spaces and the product Hardy spaces. The purpose of this paper is to extend this result to a more general class. More precisely, we introduce a class of singular integral operators including the classical Calderón-Zygmund singular integral operators and show that they are bounded on both the classical Hardy spaces and the product Hardy spaces.
\end{abstract}

\section{Introduction}

The classical Hardy spaces and the product Hardy spaces play important roles in Harmonic analysis, which are due to the original work of Fefferman and Stein [1] and Gundy and Stein [2], respectively. It is well known that these two Hardy spaces are essentially different. For instance, see [3-5]. It has been known that the classical Calderón-Zygmund singular integral operators are bounded on the classical Hardy spaces and the product singular integral operators are bounded on the product Hardy spaces. Surprisingly, in [6], we found that the classical Calderón-Zygmund singular integral operators are also bounded on the product Hardy spaces. More precisely, if $T$ is a bounded operator on $L^{2}\left(\mathbb{R}^{2}\right)$ with $T f(x)=p \cdot v$. $\mathscr{K} * f(x)$, where the kernel $\mathscr{K} \in C^{2}\left(\mathbb{R}^{2} \backslash\{0\}\right)$ and satisfies $\left|\partial_{x}^{\alpha} \mathscr{K}(x)\right| \leq C /|x|^{2+\alpha}$ for $0 \leq|\alpha| \leq 2$ and $x \in \mathbb{R}^{2} \backslash\{0\}$, then $T$ is bounded on both the classical Hardy spaces $H^{p}\left(\mathbb{R}^{2}\right)$ and the product Hardy space $H^{p}(\mathbb{R} \times \mathbb{R})$.

A natural question arises: weather there exist a more general class of operators that are bounded both on the classical Hardy spaces and the product Hardy spaces. The purpose of this paper is to answer this question. Now we first recall the definitions of the classical Hardy spaces $H^{p}\left(\mathbb{R}^{2}\right)$ (see [1] for more details) and the product Hardy spaces $H^{P}(\mathbb{R} \times \mathbb{R})($ see $[2,7]$ for more details).

We let $\mathcal{S}_{1}$ be the set including all $\psi \in \mathcal{S}\left(\mathbb{R}^{2}\right)$ that satisfy $\int_{\mathbb{R}^{2}} \psi(x) d x=0$ and $\sum_{j \in \mathbb{Z}}\left|\widehat{\psi}\left(2^{-j} \xi\right)\right|^{2}=1$ for all $\xi \in \mathbb{R}^{2} \backslash\{0\}$. And let $\mathcal{S}_{2}$ be the set including all $\tilde{\psi} \in \mathcal{S}\left(\mathbb{R}^{2}\right)$ that satisfy $\int_{\mathbb{R}} \widetilde{\psi}\left(x_{1}, x_{2}\right) d x_{1}=\int_{\mathbb{R}} \widetilde{\psi}\left(x_{1}, x_{2}\right) d x_{2}=0$ and $\sum_{j, k \in \mathbb{Z}}\left|\widehat{\widetilde{\psi}}\left(2^{-j} \xi_{1}, 2^{-k} \xi_{2}\right)\right|^{2}=1$ for all $\xi=\left(\xi_{1}, \xi_{2}\right) \in \mathbb{R}^{2} \backslash\{0\}$.

Given an $\psi \in \mathcal{S}_{1}$, the Littlewood-Paley-Stein square function of $f \in \delta^{\prime}\left(\mathbb{R}^{2}\right)$ is defined by $g_{\psi}(f)(x)=$ $\left\{\sum_{j \in \mathbb{Z}}\left|\psi_{j} * f(x)\right|^{2}\right\}^{1 / 2}$, where $\psi_{j}(x)=2^{2 j} \psi\left(2^{j} x_{1}, 2^{j} x_{2}\right)$, $x=\left(x_{1}, x_{2}\right)$. And the discrete square function is defined by $g_{\psi}^{d}(f)(x)=\left\{\sum_{j \in \mathbb{Z}} \sum_{\mathrm{Q}}\left|\psi_{j} * f\left(c_{\mathrm{Q}}\right)\right|^{2} \chi_{\mathrm{Q}}(x)\right\}^{1 / 2}$, where $\mathrm{Q}$ are dyadic cubes in $\mathbb{R}^{2}$ with the side length $l(Q)=2^{-j}$ and the center $c_{Q}$ and $\chi_{Q}$ is the characteristic function. It is well known that if $0<p<\infty$, then $\left\|g_{\psi}(f)\right\|_{L^{p}\left(\mathbb{R}^{2}\right)} \approx\left\|g_{\psi}^{d}(f)\right\|_{L^{p}\left(\mathbb{R}^{2}\right)}$ and, for different $\psi, \phi \in \mathcal{S}_{1},\left\|g_{\psi}(f)\right\|_{L^{p}\left(\mathbb{R}^{2}\right)} \approx\left\|g_{\phi}(f)\right\|_{L^{p}\left(\mathbb{R}^{2}\right)}$.

The classical Hardy space $H^{p}\left(\mathbb{R}^{2}\right)$ is then defined by

$$
H^{p}\left(\mathbb{R}^{2}\right)=\left\{f \in \mathcal{S}^{\prime} \backslash \mathscr{P}\left(\mathbb{R}^{2}\right): g_{\psi}(f)(x) \in L^{p}\left(\mathbb{R}^{2}\right)\right\},
$$

where $\mathcal{S}^{\prime} \backslash \mathscr{P}$ denotes the space of distributions modulo polynomials. The $H^{p}\left(\mathbb{R}^{2}\right)$ norm is defined by $\|f\|_{H^{p}\left(\mathbb{R}^{2}\right)}=$ $\left\|g_{\psi}(f)\right\|_{L^{p}\left(\mathbb{R}^{2}\right)}$.

Similarly, given a $\widetilde{\psi} \in \delta_{2}$, the product square function of $f \in \mathcal{S}^{\prime}\left(\mathbb{R}^{2}\right)$ is defined by $g_{\tilde{\psi}}(f)_{P}(x)=$ $\left\{\sum_{j, k \in \mathbb{Z}}\left|\widetilde{\psi}_{j, k} * f(x)\right|^{2}\right\}^{1 / 2}$, where $\widetilde{\psi}_{j, k}(x)=2^{j+k} \widetilde{\psi}\left(2^{j} x_{1}, 2^{k} x_{2}\right)$, $x=\left(x_{1}, x_{2}\right)$. And the discrete square function 
is defined by $g_{\widetilde{\psi}}^{d}(f)_{P}\left(x_{1}, x_{2}\right)=\left\{\sum_{j, k \in \mathbb{Z}} \sum_{I, J} \mid \widetilde{\psi}_{j, k} *\right.$ $\left.\left.f\left(c_{I}, c_{J}\right)\right|^{2} \chi_{I}\left(x_{1}\right) \chi_{I}\left(x_{2}\right)\right\}^{1 / 2}$, where $I, J$ are dyadic intervals in $\mathbb{R}$ with the side length $l(I)=2^{-j}, l(J)=2^{-k}$ and the center $c_{I}, c_{J}$, respectively. Also, for $0<p<\infty$, $\left\|g_{\widetilde{\psi}}(f)_{P}\right\|_{L^{p}\left(\mathbb{R}^{2}\right)} \approx\left\|g_{\widetilde{\psi}}^{d}(f)_{P}\right\|_{L^{p}\left(\mathbb{R}^{2}\right)}$ and, for different $\tilde{\psi}, \widetilde{\phi} \in \mathcal{S}_{2}$, $\left\|g_{\widetilde{\psi}}(f)_{P}\right\|_{L^{p}\left(\mathbb{R}^{2}\right)} \approx\left\|g_{\tilde{\phi}}(f)_{P}\right\|_{L^{p}\left(\mathbb{R}^{2}\right)}$.

The product Hardy space $H^{p}(\mathbb{R} \times \mathbb{R})$ is then defined by

$$
H^{p}(\mathbb{R} \times \mathbb{R})=\left\{f \in \mathcal{S}^{\prime} \backslash \mathscr{P}\left(\mathbb{R}^{2}\right): g_{\widetilde{\psi}}(f)_{P}(x) \in L^{p}\left(\mathbb{R}^{2}\right)\right\} .
$$

The $H^{p}(\mathbb{R} \times \mathbb{R})$ norm is defined by $\|f\|_{H^{p}(\mathbb{R} \times \mathbb{R})}=$ $\left\|g_{\widetilde{\psi}}(f)_{P}\right\|_{L^{p}\left(\mathbb{R}^{2}\right)}$.

The following theorem is our main result.

Theorem 1. If $2 / 3<p \leq 1, \delta>0$ and $T$ is an operator bounded on $L^{2}\left(\mathbb{R}^{2}\right)$ with $T f(x)=p \cdot v \cdot \mathscr{K} * f(x)$, where the kernel $\mathscr{K} \in C^{2}\left(\mathbb{R}^{2} \backslash\{(0, \mathbb{R}) \cup(\mathbb{R}, 0)\}\right)$ and satisfies $\left|\partial_{x_{1}}^{\alpha} \partial_{x_{2}}^{\beta} \mathscr{K}\left(x_{1}, x_{2}\right)\right| \leq C\left(1 /\left|x_{1}\right|^{1+\alpha}\right)\left(1 /\left|x_{2}\right|^{1+\beta}\right)\left(\left(\left|x_{1}\right| /\left|x_{2}\right|\right)+\right.$ $\left.\left(\left|x_{2}\right| /\left|x_{1}\right|\right)\right)^{-\delta}$ for all $|\alpha|,|\beta| \leq 1$, then $T$ is bounded on both $H^{p}\left(\mathbb{R}^{2}\right)$ and $H^{p}(\mathbb{R} \times \mathbb{R})$.

Remark 2. (I) In [8], we have shown that the operator $T$ is bounded on $L^{p}\left(\mathbb{R}^{2}\right)$ for all $1<p<\infty$.

(II) It is easy to verify that the classical CalderónZygmund singular integral operators are contained in our class. Moreover, some more operators will be in our class. For example, the operator $T=p \cdot v \cdot \mathscr{K} * f(x)$ with $\mathscr{K}\left(x_{1}, x_{2}\right)=$ $\operatorname{sgn}\left(x_{1}+x_{2}\right) /\left(\left|x_{1}\right|\left|x_{2}\right|\right)^{1 / 2}\left(\left|x_{1}\right|+\left|x_{2}\right|\right)$.

Throughout this paper, we do the following conventions.

(a) The notation $A \approx B$ means that $C_{1} A \leq B \leq C_{2} A$ for some positive constants $C_{1}, C_{2}$.

(b) If $Q$ is a cube or interval, then we denote by $c_{Q}$ its center and by $l(Q)$ its side length.

(c) For $j \in \mathbb{Z}$ and a large positive integer $N$, we denote the set $\mathscr{D}_{j}\left(\mathbb{R}^{2}\right)=\left\{Q\right.$, where $Q$ are dyadic cubes in $\mathbb{R}^{2}$ with side length $\left.l(Q)=2^{-j}\right\}$ and $\mathscr{D}_{j}^{N}\left(\mathbb{R}^{2}\right)=\mathscr{D}_{j+N}\left(\mathbb{R}^{2}\right)$.

(d) For $j, k \in \mathbb{Z}$ and a large positive integer $N$, we denote the set $\mathscr{D}_{j, k}\left(\mathbb{R}^{2}\right)=\{R=I \times J$, where $I$ and $J$ are dyadic intervals in $\mathbb{R}$ with side length $l(I)=2^{-j}$ and $l(J)=$ $2^{-k}$, respectively $\}$ and $\mathscr{D}_{j, k}^{N}\left(\mathbb{R}^{2}\right)=\mathscr{D}_{j+N, k+N}\left(\mathbb{R}^{2}\right)$.

(e) $j \wedge j^{\prime}$ means the minimum of $j$ and $j^{\prime}$.

\section{Proof of Theorem 1}

The first crucial tool in the proof of Theorem 1 is to apply the following discrete Calderón identity (see [9] for more details).

Lemma 3. If $0<p \leq 1$, then consider the following.

(a) Suppose that $\phi \in \mathcal{S}_{1} \cap C_{0}^{\infty}\left(\mathbb{R}^{2}\right)$ with $\operatorname{supp}(\phi) \subset\{x$ : $|x| \leq 1\}$. Then for all $f \in L^{2}\left(\mathbb{R}^{2}\right) \cap H^{p}\left(\mathbb{R}^{2}\right)$, there exist a large positive integer $N$ (depending only on $p$ ) and a function $h \in$
$L^{2}\left(\mathbb{R}^{2}\right) \cap H^{P}\left(\mathbb{R}^{2}\right)$ such that $f(x)=\sum_{j \in \mathbb{Z}} \sum_{\mathrm{Q} \in \mathscr{D}_{j}^{N}\left(\mathbb{R}^{2}\right)}|Q| \phi_{j}(x-$ $\left.c_{Q}\right)\left(\phi_{j} * h\right)\left(c_{Q}\right)$, where the series converges in $L^{2}\left(\mathbb{R}^{2}\right)$. Moreover, $\|f\|_{L^{2}\left(\mathbb{R}^{2}\right)} \approx\|h\|_{L^{2}\left(\mathbb{R}^{2}\right)}$ and $\|f\|_{H^{p}\left(\mathbb{R}^{2}\right)} \approx\|h\|_{L^{2}\left(\mathbb{R}^{2}\right)}$.

(b) Suppose that $\tilde{\phi} \in \mathcal{S}_{2} \cap C_{0}^{\infty}\left(\mathbb{R}^{2}\right)$ with $\operatorname{supp}(\widetilde{\phi}) \quad \subset$ $\{x:|x| \leq 1\}$. Then for all $f \in L^{2}\left(\mathbb{R}^{2}\right) \cap H^{p}(\mathbb{R} \times \mathbb{R})$, there exist a large positive integer $N$ (depending only on $p$ ) and a function $h \in L^{2}\left(\mathbb{R}^{2}\right) \cap H^{p}(\mathbb{R} \times \mathbb{R})$ such that $f(x)=$ $\sum_{j, k \in \mathbb{Z}} \sum_{R \in \mathscr{D}_{j, k}^{N}\left(\mathbb{R}^{2}\right)}|R| \phi_{j, k}\left(x-c_{R}\right)\left(\phi_{j, k} * h\right)\left(c_{R}\right)$, where the series converges in $L^{2}\left(\mathbb{R}^{2}\right)$. Moreover, $\|f\|_{H^{p}\left(\mathbb{R}^{2}\right)} \approx\|h\|_{L^{2}\left(\mathbb{R}^{2}\right)}$ and $\|f\|_{H^{p}(\mathbb{R} \times \mathbb{R})} \approx\|h\|_{H^{p}(\mathbb{R} \times \mathbb{R})_{P}}$.

For the proof, we refer readers to [9].

The second crucial tool in the proof of Theorem 1 is the following orthogonal estimates.

Lemma 4. Suppose that $0<\lambda \leq \min (\delta, 1 / 2)$ and $\mathscr{K}$ is the kernel as in Theorem 1; then

(a) for $\phi \in C_{0}^{\infty}\left(\mathbb{R}^{2}\right)$ with $\int_{\mathbb{R}^{2}} \phi(x) d x=0$, one has $\mid \mathscr{K} *$ $\phi_{j}(x) \mid \leq C 2^{2 j}\left(1 /\left(1+\left|2^{j} x_{1}\right|^{1+\lambda}\right)\right)\left(1 /\left(1+\left|2^{j} x_{2}\right|^{1+\lambda}\right)\right)$, for all $x=$ $\left(x_{1}, x_{2}\right) \in \mathbb{R}^{2}$ and $j \in \mathbb{Z}$, where $C$ is a constant independent of $j$ and $x$;

(b) for $\tilde{\phi} \in C_{0}^{\infty}\left(\mathbb{R}^{2}\right)$ with $\int_{\mathbb{R}} \tilde{\phi}\left(x_{1}, x_{2}\right) d x_{1}=$ $\int_{\mathbb{R}} \tilde{\phi}\left(x_{1}, x_{2}\right) d x_{2}=0$, one has $\left|\mathscr{K} * \tilde{\phi}_{j, k}(x)\right| \leq$ $C 2^{j+k}\left(1 /\left(1+\left|2^{j} x_{1}\right|^{1+\lambda}\right)\right)\left(1 /\left(1+\left|2^{k} x_{2}\right|^{1+\lambda}\right)\right)$, for all $x=\left(x_{1}, x_{2}\right) \in \mathbb{R}^{2}$ and $j, k \in \mathbb{Z}$, where $C$ is a constant independent of $j, k$, and $x$.

Proof. (a) Since $\mathscr{K}$ is single-parameter dilation invariant, that is, for each $\delta>0, \delta^{2} \mathscr{K}\left(\delta x_{1}, \delta x_{2}\right)$ satisfies the same hypotheses, with the same bounds as $\mathscr{K}$. We just need to show that $|\mathscr{K} * \phi(x)| \leq C\left(\left(1 /\left(1+\left|x_{1}\right|^{1+\lambda}\right)\right)\right)\left(1 /\left(1+\left|x_{2}\right|^{1+\lambda}\right)\right)$, for all $x=\left(x_{1}, x_{2}\right) \in \mathbb{R}^{2}$. Without loss of generality, we may assume that $\operatorname{supp}(\phi) \subset\{x:|x| \leq 1\}$. To get the required estimate, we will discuss it in the following three cases: (I) $\left|x_{1}\right| \geq 2,\left|x_{2}\right| \geq 2$; (II) $\left|x_{1}\right| \geq 2,\left|x_{2}\right|<2$ or $\left|x_{1}\right|<2,\left|x_{2}\right| \geq 2$; (III) $\left|x_{1}\right|<2,\left|x_{2}\right|<2$.

For case (I), $\left|x_{1}\right| \geq 2,\left|x_{2}\right| \geq 2$, by the moment condition of $\phi$, we have

$$
\begin{aligned}
& |\mathscr{K} * \phi(x)| \\
& =\left|\int_{\mathbb{R}^{2}} \mathscr{K}\left(x_{1}-y_{1}, x_{2}-y_{2}\right) \phi\left(y_{1}, y_{2}\right) d y_{1} d y_{2}\right| \\
& =\mid \int_{\mathbb{R}^{2}}\left(\mathscr{K}\left(x_{1}-y_{1}, x_{2}-y_{2}\right)-\mathscr{K}\left(x_{1}, x_{2}\right)\right) \\
& \quad \times \phi\left(y_{1}, y_{2}\right) d y_{1} d y_{2} \mid \\
& \leq C \mid \int_{\mathbb{R}^{2}}\left(\frac{1}{\left|x_{1}\right|^{2}} \frac{1}{\left|x_{2}\right|}+\frac{1}{\left|x_{1}\right|} \frac{1}{\left|x_{2}\right|^{2}}\right)\left(\frac{\left|x_{1}\right|}{\left|x_{2}\right|}+\frac{\left|x_{2}\right|}{\left|x_{1}\right|}\right)^{-\delta} \\
& \quad \times\left|\phi\left(y_{1}, y_{2}\right)\right| d y_{1} d y_{2} \mid
\end{aligned}
$$




$$
\begin{aligned}
& \leq C\left(\frac{1}{\left|x_{1}\right|^{2}} \frac{1}{\left|x_{2}\right|}+\frac{1}{\left|x_{1}\right|} \frac{1}{\left|x_{2}\right|^{2}}\right)\left(\frac{\left|x_{1}\right|}{\left|x_{2}\right|}+\frac{\left|x_{2}\right|}{\left|x_{1}\right|}\right)^{-\delta} \\
& \leq \frac{C}{1+\left|x_{1}\right|^{1+\lambda}} \frac{1}{1+\left|x_{2}\right|^{1+\lambda}} .
\end{aligned}
$$
have

For case (II), $\left|x_{1}\right| \geq 2,\left|x_{2}\right|<2$ or $\left|x_{1}\right|<2,\left|x_{2}\right| \geq 2$, we

$$
\begin{aligned}
\mid \mathscr{K} * & \phi(x) \mid \\
& \leq \int_{\mathbb{R}^{2}}\left|\mathscr{K}\left(x_{1}-y_{1}, x_{2}-y_{2}\right) \phi\left(y_{1}, y_{2}\right)\right| d y_{1} d y_{2} \\
& \leq C \int_{\left|y_{1}\right| \leq 1} \frac{1}{\left|y_{2}\right| \leq 1}\left|\frac{1}{\left|x_{1}-y_{1}\right|}\right| y_{2} \mid \\
& \times\left(\frac{\left|x_{1}-y_{1}\right|}{\left|x_{2}-y_{2}\right|}+\frac{\left|x_{2}-y_{2}\right|}{\left|x_{1}-x_{2}\right|}\right)^{-\delta} d y_{1} d y_{2} \\
& \leq \frac{C}{1+\left|x_{1}\right|^{1+\delta}} \frac{1}{1+\left|x_{2}\right|^{1+\delta}} \leq \frac{C}{1+\left|x_{1}\right|^{1+\lambda}} \frac{1}{1+\left|x_{2}\right|^{1+\lambda}} .
\end{aligned}
$$

For case (III), $\left|x_{1}\right|<2,\left|x_{2}\right|<2$, we let $\eta \in C_{0}^{\infty}\left(\mathbb{R}^{2}\right)$ with $0 \leq \eta(x) \leq 1$ and $\eta(x)=1$ when $|x| \leq 4$ and $\eta(x)=0$ when $|x| \geq 8$. We have

$$
\begin{aligned}
& |\mathscr{K} * \phi(x)| \\
& =\left|\int_{\mathbb{R}^{2}} \mathscr{K}\left(y_{1}, y_{2}\right) \phi\left(x_{1}-y_{1}, x_{2}-y_{2}\right) \eta\left(y_{1}, y_{2}\right) d y_{1} d y_{2}\right| \\
& \leq \mid \int_{\mathbb{R}^{2}} \mathscr{K}\left(y_{1}, y_{2}\right)\left(\phi\left(x_{1}-y_{1}, x_{2}-y_{2}\right)-\phi\left(x_{1}, x_{2}\right)\right) \\
& \quad \times \eta\left(y_{1}, y_{2}\right) d y_{1} d y_{2} \mid \\
& \quad+\left|\int_{\mathbb{R}^{2}} \mathscr{K}\left(y_{1}, y_{2}\right) \phi\left(x_{1}, x_{2}\right) \eta\left(y_{1}, y_{2}\right) d y_{1} d y_{2}\right| \\
& \leq C \mid \int_{\left|y_{1}\right| \leq 8} \frac{1}{\left|y_{2}\right| \leq 8} \frac{1}{\left|y_{2}\right|}\left(\frac{\left|y_{2}\right|}{\left|y_{1}\right|}+\frac{\left|y_{1}\right|}{\left|y_{2}\right|}\right)^{-\delta} \\
& \quad \times\left(\left|y_{1}\right|+\left|y_{2}\right|\right) d y_{1} d y_{2} \mid \\
& \quad+C\left|\int_{\mathbb{R}^{2}} \widetilde{\mathscr{K}}\left(\xi_{1}, \xi_{2}\right) \widetilde{\eta}\left(\xi_{1}, \xi_{2}\right) d y_{1} d y_{2}\right| \\
& \leq C \leq \frac{C}{1+\left|x_{1}\right|^{1+\lambda}} \frac{1}{1+\left|x_{2}\right|^{1+\lambda}} .
\end{aligned}
$$

(b) Without loss of generality, we may assume that $\operatorname{supp}(\widetilde{\phi}) \subset\{x:|x| \leq 1\}$. The required estimate will be discussed in the following four cases: (I) $\left|x_{1}\right| \geq 2^{-j+1},\left|x_{2}\right| \geq$ $2^{-k+1}$; (II) $\left|x_{1}\right| \geq 2^{-j+1},\left|x_{2}\right|<2^{-k+1}$; (III) $\left|x_{1}\right|<2^{-j+1},\left|x_{2}\right| \geq$ $2^{-k+1}$; (IV) $\left|x_{1}\right|<2^{-j+1},\left|x_{2}\right|<2^{-k+1}$.

For case (I), $\left|x_{1}\right| \geq 2^{-j+1},\left|x_{2}\right| \geq 2^{-k+1}$, by the moment condition of $\widetilde{\phi}$, we have

$$
\begin{aligned}
& \left|\mathscr{K} * \widetilde{\phi}_{j, k}(x)\right| \\
& =2^{j+k} \mid \int_{\mathbb{R}^{2}}\left\{\left(\mathscr{K}\left(x_{1}-y_{1}, x_{2}-y_{2}\right)-\mathscr{K}\left(x_{1}, x_{2}-y_{2}\right)\right)\right. \\
& \left.-\left(\mathscr{K}\left(x_{1}-y_{1}, x_{2}\right)-\mathscr{K}\left(x_{1}, x_{2}\right)\right)\right\} \\
& \times \widetilde{\phi}\left(2^{j} y_{1}, 2^{k} y_{2}\right) d y_{1} d y_{2} \\
& =2^{j+k} \mid \int_{\mathbb{R}^{2}} \int_{x_{2}}^{x_{2}-y_{2}} \int_{x_{1}}^{x_{1}-y_{1}} \partial_{z_{1}}^{1} \partial_{z_{2}}^{1} \mathscr{K}\left(z_{1}, z_{2}\right) d z_{1} d z_{2} \\
& \times \widetilde{\phi}\left(2^{j} y_{1}, 2^{k} y_{2}\right) d y_{1} d y_{2} \\
& \leq C 2^{j+k} \int_{\mathbb{R}^{2}} \frac{1}{\left|x_{1}\right|^{2}} \frac{1}{\left|x_{2}\right|^{2}}\left(\frac{\left|x_{1}\right|}{\left|x_{2}\right|}+\frac{\left|x_{2}\right|}{\left|x_{1}\right|}\right)^{-\delta} \\
& \times\left|y_{1}\right|\left|y_{2}\right|\left|\widetilde{\phi}\left(2^{j} y_{1}, 2^{k} y_{2}\right)\right| d y_{1} d y_{2} \\
& \leq C 2^{-j-k} \frac{1}{\left|x_{1}\right|^{2}} \frac{1}{\left|x_{2}\right|^{2}} \leq C 2^{j+k} \frac{1}{1+\left|2^{j} x_{1}\right|^{1+\lambda}} \frac{1}{1+\left|2^{k} x_{2}\right|^{1+\lambda}} \text {. }
\end{aligned}
$$

For case (II), $\left|x_{1}\right| \geq 2^{-j+1},\left|x_{2}\right|<2^{-k+1}$, similarly, we have

$$
\begin{aligned}
& \left|\mathscr{K} * \widetilde{\phi}_{j, k}(x)\right| \\
& \begin{array}{l}
=2^{j+k} \mid \int_{\mathbb{R}^{2}}\left(\mathscr{K}\left(x_{1}-y_{1}, x_{2}-y_{2}\right)-\mathscr{K}\left(x_{1}, x_{2}-y_{2}\right)\right) \\
\quad \times \tilde{\phi}\left(2^{j} y_{1}, 2^{k} y_{2}\right) d y_{1} d y_{2} \mid \\
=2^{j+k} \mid \int_{\mathbb{R}^{2}} \int_{x_{1}}^{x_{1}-y_{1}} \partial_{z_{1}}^{1} \mathscr{K}\left(z_{1}, x_{2}-y_{2}\right) d z_{1} \\
\quad \times \widetilde{\phi}\left(2^{j} y_{1}, 2^{k} y_{2}\right) d y_{1} d y_{2} \mid \\
\leq C 2^{j+k} \int_{\left|y_{1}\right| \leq 2^{-j} \frac{1}{\left|y_{2}\right| \leq 2^{-k}} \frac{1}{\left|x_{1}\right|^{2}}\left|x_{2}-y_{2}\right|} \\
\quad \times\left(\frac{\left|x_{1}\right|}{\left|x_{2}-y_{2}\right|}+\frac{\left|x_{2}-y_{2}\right|}{\left|x_{1}\right|}\right)^{-\delta}\left|y_{1}\right| d y_{1} d y_{2}
\end{array} \\
& \leq C 2^{-j+k} \frac{1}{\left|x_{1}\right|^{2}} \leq C 2^{j+k} \frac{1}{1+\left|2^{j} x_{1}\right|^{1+\lambda}} \frac{1}{1+\left|2^{k} x_{2}\right|^{1+\lambda}} .
\end{aligned}
$$

The cases (II) and (III) are symmetric, so case (III) follows. 
For case (IV), $\left|x_{1}\right|<2^{-j+1},\left|x_{2}\right|<2^{-k+1}$, we let $\theta \in C_{0}^{\infty}(\mathbb{R})$ with $0 \leq \theta(x) \leq 1$ and $\theta(x)=1$ when $|x| \leq 4$ and $\theta(x)=0$ when $|x| \geq 8$. Then

$$
\begin{aligned}
& \left|\mathscr{K} * \widetilde{\phi}_{j, k}(x)\right| \\
& =2^{j+k} \mid \int_{\mathbb{R}^{2}} \mathscr{K}\left(y_{1}, y_{2}\right) \tilde{\phi}\left(2^{j}\left(x_{1}-y_{1}\right), 2^{k}\left(x_{2}-y_{2}\right)\right) \\
& \times \theta\left(2^{j} y_{1}\right) \theta\left(2^{k} y_{2}\right) d y_{1} d y_{2} \\
& \leq 2^{j+k} \mid \int_{\mathbb{R}^{2}} \mathscr{K}\left(y_{1}, y_{2}\right) \\
& \times\left(\widetilde{\phi}\left(2^{j}\left(x_{1}-y_{1}\right), 2^{k}\left(x_{2}-y_{2}\right)\right)-\widetilde{\phi}\right. \\
& \left.\times\left(2^{j} x_{1}, 2^{k} x_{2}\right)\right) \theta\left(2^{j} y_{1}\right) \theta\left(2^{k} y_{2}\right) d y_{1} d y_{2} \\
& +2^{j+k} \mid \int_{\mathbb{R}^{2}} \mathscr{K}\left(y_{1}, y_{2}\right) \tilde{\phi}\left(2^{j} x_{1}, 2^{k} x_{2}\right) \\
& \times \theta\left(2^{j} y_{1}\right) \theta\left(2^{k} y_{2}\right) d y_{1} d y_{2} \\
& \leq C 2^{j+k} \int_{\substack{\left|y_{1}\right| \leq 2^{-j+3}\left|\leq 2^{-k+3}\\
\right| y_{2}\left|\leq y_{1}\right|}} \frac{1}{\left|y_{2}\right|}\left(\frac{\left|y_{1}\right|}{\left|y_{2}\right|}+\frac{\left|y_{2}\right|}{\left|y_{1}\right|}\right)^{-\delta} \\
& \times\left(\left|2^{j} y_{1}\right|+\left|2^{k} y_{2}\right|\right) d y_{1} d y_{2} \\
& +C\left|\int_{\mathbb{R}^{2}} \widehat{\mathscr{K}}\left(\xi_{1}, \xi_{2}\right) \hat{\theta}\left(2^{-j} \xi_{1}\right) \hat{\theta}\left(2^{-k} \xi_{2}\right) d \xi_{1} d \xi_{2}\right| \\
& \leq C 2^{j+k} \leq C 2^{j+k} \frac{1}{1+\left|2^{j} x_{1}\right|^{1+\lambda}} \frac{1}{1+\left|2^{k} x_{2}\right|^{1+\lambda}} .
\end{aligned}
$$

This completes the proof of Lemma 4.

As a consequence of Lemma 4, we have the following.

Lemma 5. (a) Under hypothesis (a) of Lemma 4, one has

$$
\begin{aligned}
\left|\phi_{j} * \mathscr{K} * \phi_{j^{\prime}}(x)\right| \leq & C 2^{-\left|j-j^{\prime}\right|} \frac{2^{j \wedge j^{\prime}}}{1+\left|2^{j \wedge j^{\prime}} x_{1}\right|^{1+\lambda}} \\
& \times \frac{2^{j \wedge j^{\prime}}}{1+\left|2^{j \wedge j^{\prime}} x_{2}\right|^{1+\lambda}},
\end{aligned}
$$

$$
\times \frac{2^{k \wedge k^{\prime}}}{1+\left|2^{k \wedge k^{\prime}} x_{2}\right|^{1+\lambda}},
$$

for all $j, j^{\prime}, k$ and $k^{\prime} \in \mathbb{Z}$ and $x=\left(x_{1}, x_{2}\right) \in \mathbb{R}^{2}$.
The proof of Lemma 5 is based on the following two observations: (1) convolution operation is commutative; that is, $\phi_{j} * \mathscr{K} * \phi_{j^{\prime}}(x)=\mathscr{K} *\left(\phi_{j} * \phi_{j^{\prime}}\right)(x)\left(\right.$ or $\widetilde{\phi}_{j, k} * \mathscr{K} * \widetilde{\phi}_{j^{\prime}, k^{\prime}}(x)=$ $\left.\mathscr{K} *\left(\widetilde{\phi}_{j, k} * \widetilde{\phi}_{j^{\prime}, k^{\prime}}\right)(x)\right) ;(2) \phi_{j} * \phi_{j^{\prime}}\left(\right.$ or $\left.\widetilde{\phi}_{j, k} * \widetilde{\phi}_{j^{\prime}, k^{\prime}}\right)$ satisfies the same estimate as $\phi_{j \wedge j^{\prime}}\left(\right.$ or $\left.\widetilde{\phi}_{j \wedge j^{\prime}, k \wedge k^{\prime}}\right)$ in Lemma 4 with the bound $C 2^{-\left|j-j^{\prime}\right|}$ (or $C 2^{-\left|j-j^{\prime}\right|} 2^{-\left|k-k^{\prime}\right|}$ ). The details are left to the readers.

The last crucial tool in the proof of Theorem 1 is the following strongly maximal function estimates.

Lemma 6. Suppose that $\lambda>0,2 / 3<q \leq 1, N, j, j^{\prime}, k$ and $k^{\prime} \in \mathbb{Z}$, and $F \in L^{2}\left(\mathbb{R}^{2}\right)$. Then consider the following.

(a) If $Q^{\prime} \in \mathscr{D}_{j^{\prime}}\left(\mathbb{R}^{2}\right), u=\left(u_{1}, u_{2}\right)$, and $v=\left(v_{1}, v_{2}\right) \in Q^{\prime}$, then one has

$$
\begin{aligned}
& \sum_{Q=I \times J \in \mathscr{D}_{j}^{N}\left(\mathbb{R}^{2}\right)} \frac{2^{j \wedge j^{\prime}}}{\left(1+2^{j \wedge j^{\prime}}\left|u_{1}-c_{I}\right|\right)^{1+\lambda}} \frac{2^{j \wedge j^{\prime}}}{\left(1+2^{j \wedge j^{\prime}}\left|u_{2}-c_{J}\right|\right)^{1+\lambda}} \\
& \times\left|F\left(c_{\mathrm{Q}}\right)\right| \\
& \leq C 2^{2\left(j \wedge j^{\prime}\right)(1-1 / q)+2 j / q} \\
& \times\left\{M_{S}\left[\left(\sum_{Q \in \mathscr{D}_{j}^{N}\left(\mathbb{R}^{2}\right)}\left|F\left(c_{Q}\right)\right|^{2} \chi_{\mathrm{Q}}\right)^{q / 2}\right]\right\}^{1 / q}(v),
\end{aligned}
$$

where $M_{S}$ is the strongly maximal operator.

(b) If $R^{\prime}=I^{\prime} \times J^{\prime} \in \mathscr{D}_{j^{\prime}, k^{\prime}}\left(\mathbb{R}^{2}\right), u=\left(u_{1}, u_{2}\right)$, and $v=$ $\left(v_{1}, v_{2}\right) \in R^{\prime}$, then one has

$$
\begin{aligned}
\sum_{R=I \times J \in \mathscr{D}_{j, k}^{N}\left(\mathbb{R}^{2}\right)} \frac{2^{j \wedge j^{\prime}}}{\left(1+2^{j \wedge j^{\prime}}\left|u_{1}-c_{I}\right|\right)^{1+\lambda}} \frac{2^{k \wedge k^{\prime}}}{\left(1+2^{k \wedge k^{\prime}}\left|u_{2}-c_{J}\right|\right)^{1+\lambda}} \\
\times\left|F\left(c_{I}, c_{J}\right)\right| \\
\leq C 2^{\left(j \wedge j^{\prime}\right)(1-1 / q)+j / q} 2^{\left(k \wedge k^{\prime}\right)(1-1 / q)+k / q} \\
\times\left\{M_{S}\left[\left(\sum_{R \in \mathscr{D}_{j, k}^{N}\left(\mathbb{R}^{2}\right)}\left|F\left(c_{R}\right)\right|^{2} \chi_{R}\right)^{q / 2}\right]\right\}^{1 / q}(v) .
\end{aligned}
$$

For the proof, we refer readers to [10].

Proof of Theorem 1. Firstly we show that $T$ is bounded on the classical Hardy space $H^{p}\left(\mathbb{R}^{2}\right)$. Since $L^{2}\left(\mathbb{R}^{2}\right) \cap H^{p}\left(\mathbb{R}^{2}\right)$ is dense in $H^{p}\left(\mathbb{R}^{2}\right)$, we just need to show that, for all $f \in$ $L^{2}\left(\mathbb{R}^{2}\right) \cap H^{p}\left(\mathbb{R}^{2}\right)$, we have $\|T f\|_{H^{p}\left(\mathbb{R}^{2}\right)} \leq C\|f\|_{H^{p}\left(\mathbb{R}^{2}\right)}$; that is, 
for a fixed $\psi \in \mathcal{S}_{1}$,

$$
\left\|g_{\psi}(T f)\right\|_{L^{p}\left(\mathbb{R}^{2}\right)} \leq C\|f\|_{H^{p}\left(\mathbb{R}^{2}\right)} .
$$

Note that

$g_{\psi}(T f)(x)$

$=\left\{\sum_{j^{\prime} \in \mathbb{Z} Q^{\prime}=I^{\prime} \times J^{\prime} \in \mathscr{D}_{j^{\prime}}\left(\mathbb{R}^{2}\right)}\left|\psi_{j^{\prime}} * \mathscr{K} * f\left(c_{Q^{\prime}}\right)\right|^{2} \chi_{Q^{\prime}}(x)\right\}^{1 / 2}$.

For $j^{\prime} \in \mathbb{Z}, Q^{\prime}=I^{\prime} \times J^{\prime} \in \mathscr{D}_{j^{\prime}}\left(\mathbb{R}^{2}\right)$, and $x \in Q^{\prime}$, applying (a) of Lemma 3, we have

$$
\begin{aligned}
& \left|\psi_{j^{\prime}} * \mathscr{K} * f\left(c_{Q^{\prime}}\right)\right| \\
& =\left|\sum_{j \in \mathbb{Z}} \sum_{\mathrm{Q} \in \mathscr{D}_{j}^{N}\left(\mathbb{R}^{2}\right)}\right| Q\left|\psi_{j^{\prime}} * \mathscr{K} * \psi_{j}\left(u-c_{\mathrm{Q}}\right)\left(\psi_{j} * h\right)\left(c_{\mathrm{Q}}\right)\right| .
\end{aligned}
$$

By (a) of Lemma 5, we have

$$
\begin{aligned}
& \left|\psi_{j^{\prime}} * \mathscr{K} * f\left(c_{Q^{\prime}}\right)\right| \\
& \leq C \sum_{j \in \mathbb{Z} Q=I \times J \in \mathscr{D}_{j^{\prime}}\left(\mathbb{R}^{2}\right)} 2^{-2 j} 2^{-\left|j-j^{\prime}\right|} \\
& \times \frac{2^{j \wedge j^{\prime}}}{1+\left|2^{j \wedge j^{\prime}}\left(u_{1}-c_{I}\right)\right|^{1+\lambda}} \\
& \times \frac{2^{j \wedge j^{\prime}}}{1+\left|2^{j \wedge j^{\prime}}\left(u_{2}-c_{J}\right)\right|^{1+\lambda}}\left(\psi_{j} * h\right)\left(c_{Q}\right) \text {. }
\end{aligned}
$$

Applying (a) of Lemma 6 with $F=\psi_{j} * h$ and $2 / 3<q<p$, we have

$$
\begin{aligned}
& \left|\psi_{j^{\prime}} * \mathscr{K} * f\left(c_{Q^{\prime}}\right)\right| \\
& \quad \leq C \sum_{j \in \mathbb{Z}} 2^{-2 j} 2^{-\left|j-j^{\prime}\right|} 2^{2\left(j \wedge j^{\prime}\right)(1-1 / q)+2 j / q} \\
& \quad \times\left\{M_{S}\left[\left(\sum_{Q \in \mathscr{D}_{j}^{N}\left(\mathbb{R}^{2}\right)}\left|\psi_{j} * h\left(c_{\mathrm{Q}}\right)\right|^{2} \chi_{\mathrm{Q}}\right)^{q / 2}\right]\right\}^{1 / q}(x) .
\end{aligned}
$$

Therefore,

$$
\begin{aligned}
& \left|g_{\psi}(T f)(x)\right|^{2}=\sum_{j^{\prime} \in \mathbb{Z} Q_{Q^{\prime} \in \mathscr{D}_{j^{\prime}}\left(\mathbb{R}^{2}\right)}}\left|\psi_{j^{\prime}} * \mathscr{K} * f\left(c_{\mathrm{Q}^{\prime}}\right)\right|^{2} \chi_{\mathrm{Q}^{\prime}}(x) \\
& \leq C \sum_{j^{\prime} \in \mathbb{Z} Q^{\prime} \in \mathscr{D}_{j^{\prime}}\left(\mathbb{R}^{2}\right)} \mid \sum_{j \in \mathbb{Z}} 2^{-2 j} 2^{-\left|j-j^{\prime}\right|} 2^{2\left(j \wedge j^{\prime}\right)(1-1 / q)+2 j / q} \\
& \times\left.\left\{\left[\left(\sum_{Q \in D_{j}^{N}\left(\mathbb{R}^{2}\right)}\left|\psi_{j} * h\left(c_{\mathrm{Q}}\right)\right|^{2} \chi_{\mathrm{Q}}\right)^{q / 2}\right]\right\}^{1 / q}(x)\right|^{2} \chi_{\mathrm{Q}^{\prime}}(x) \\
& \leq C \sum_{j^{\prime} \in \mathbb{Z}}\left(\sum_{j \in \mathbb{Z}} 2^{-2 j} 2^{-\left|j-j^{\prime}\right|} 2^{2\left(j \wedge j^{\prime}\right)(1-1 / q)+2 j / q}\right) \\
& \times\left(\sum_{j \in \mathbb{Z}} 2^{-2 j} 2^{-\left|j-j^{\prime}\right|} 2^{2\left(j \wedge j^{\prime}\right)(1-1 / q)+2 j / q}\left\{M_{S}\left[\left(\sum_{Q \in \mathscr{D}_{j}^{N}\left(\mathbb{R}^{2}\right)}\left|\psi_{j} * h\left(c_{Q}\right)\right|^{2} \chi_{\mathrm{Q}}\right)^{q / 2}\right]\right\}^{2 / q}(x)\right)
\end{aligned}
$$




$$
\begin{aligned}
& \leq C \sum_{j^{\prime} \in \mathbb{Z}} \sum_{j \in \mathbb{Z}} 2^{-2 j} 2^{-\left|j-j^{\prime}\right|} 2^{2\left(j \wedge j^{\prime}\right)(1-1 / q)+2 j / q}\left\{M_{S}\left[\left(\sum_{Q \in \mathscr{D}_{j}^{N}\left(\mathbb{R}^{2}\right)}\left|\psi_{j} * h\left(c_{\mathrm{Q}}\right)\right|^{2} \chi_{\mathrm{Q}}\right)^{q / 2}\right]\right\}^{2 / q}(x) \\
& \leq C \sum_{j \in \mathbb{Z}}\left\{M_{S}\left[\left(\sum_{Q \in \mathscr{D}_{j}^{N}\left(\mathbb{R}^{2}\right)}\left|\psi_{j} * h\left(c_{\mathrm{Q}}\right)\right|^{2} \chi_{\mathrm{Q}}\right)^{q / 2}\right]\right\}^{2 / q}(x) .
\end{aligned}
$$

Applying Fefferman-Stein's vector-valued strong maximal inequality (see [11] for more details) on $L^{p / q}\left(\ell^{2 / q}\right)$, we have

$$
\begin{aligned}
\|T(f)\|_{H^{p}\left(\mathbb{R}^{2}\right)} & =\left\|g_{\psi}(T f)\right\|_{L^{p}\left(\mathbb{R}^{2}\right)} \\
& \leq C\left\|\left\{\sum_{j \in \mathbb{Z}}\left\{M_{S}\left[\left(\sum_{Q \in \mathscr{D}_{j}^{N}\left(\mathbb{R}^{2}\right)}\left|\psi_{j} * h\left(c_{\mathrm{Q}}\right)\right|^{2} \chi_{\mathrm{Q}}\right)^{q / 2}\right]\right\}^{2 / q}(x)\right\}^{1 / 2}\right\|_{L^{p}\left(\mathbb{R}^{2}\right)} \\
& \leq C\left\|\left\{\sum_{j \in \mathbb{Z}} \sum_{\mathrm{Q} \in \mathscr{D}_{j}^{N}\left(\mathbb{R}^{2}\right)}\left|\psi_{j} * h\left(c_{\mathrm{Q}}\right)\right|^{2} \chi_{\mathrm{Q}}(x)\right\}^{1 / 2}\right\|_{L^{p}\left(\mathbb{R}^{2}\right)} \\
& \leq C\|h\|_{H^{p}\left(\mathbb{R}^{2}\right)} \leq C\|f\|_{H^{p}\left(\mathbb{R}^{2}\right)}
\end{aligned}
$$

The proof of T's boundedness on $H^{p}(\mathbb{R} \times \mathbb{R})$ is almost the same as above; that is, we just need to replace (a) of the required lemmas to (b). Here we omit the details.

\section{Conflict of Interests}

The author declares that there is no conflict of interests regarding the publication of this paper.

\section{Acknowledgment}

Chaoqiang Tan is supported by SRF for the Doctoral Program of Higher Education (Grant no. 20104402120002).

\section{References}

[1] C. Fefferman and E. M. Stein, " $H^{p}$ spaces of several variables," Acta Mathematica, vol. 129, no. 3-4, pp. 137-193, 1972.

[2] R. F. Gundy and E. M. Stein, " $H^{p}$ theory for the poly-disc," Proceedings of the National Academy of Sciences of the United States of America, vol. 76, no. 3, pp. 1026-1029, 1979.
[3] L. Carleson, "A counterexample for measures bounded on $\mathrm{Hp}$ for the bi-disc," Mittag Leffler Report 7, 1974.

[4] S. Y. Chang and R. Fefferman, "A continuous version of duality of $H^{1}$ with BMO on the bidisc," Annals of Mathematics, vol. 112, no. 1, pp. 179-201, 1980.

[5] S. Y. Chang and R. Fefferman, "Some recent developments in Fourier analysis and $H^{p}$-theory on product domains," Bulletin of the American Mathematical Society, vol. 12, no. 1, pp. 1-43, 1985.

[6] C. Q. Tan, "Boundedness of classical Calderón-Zygmund convolution operators on product Hardy space," Mathematical Research Letters, vol. 20, no. 3, pp. 591-599, 2013.

[7] R. Fefferman, "Calderón-Zygmund theory for product domains: $H^{p}$ spaces," Proceedings of the National Academy of Sciences of the United States of America, vol. 83, no. 4, pp. 840-843, 1986.

[8] C. Q. Tan, "Singular integral operators between the classical type and the product type," Acta Scientiarum Naturalium Universitatis Sunyatseni, vol. 51, no. 5, pp. 58-62, 2012.

[9] Y. S. Han, G. Z. Lu, and K. Zhao, "Discrete Calderón's identity, atomic decomposition and boundedness criterion of operators 
on multiparameter hardy spaces," Journal of Geometric Analysis, vol. 20, no. 3, pp. 670-689, 2010.

[10] Y.-S. Han, "Plancherel-Pólya type inequality on spaces of homogeneous type and its applications," Proceedings of the American Mathematical Society, vol. 126, no. 11, pp. 3315-3327, 1998.

[11] E. M. Stein, "Note on singular integrals," Proceedings of the American Mathematical Society, vol. 8, pp. 250-254, 1957. 


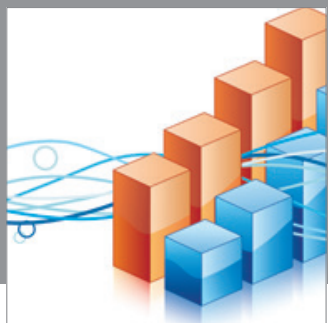

Advances in

Operations Research

mansans

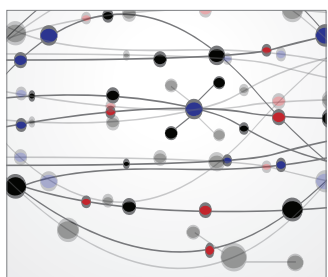

The Scientific World Journal
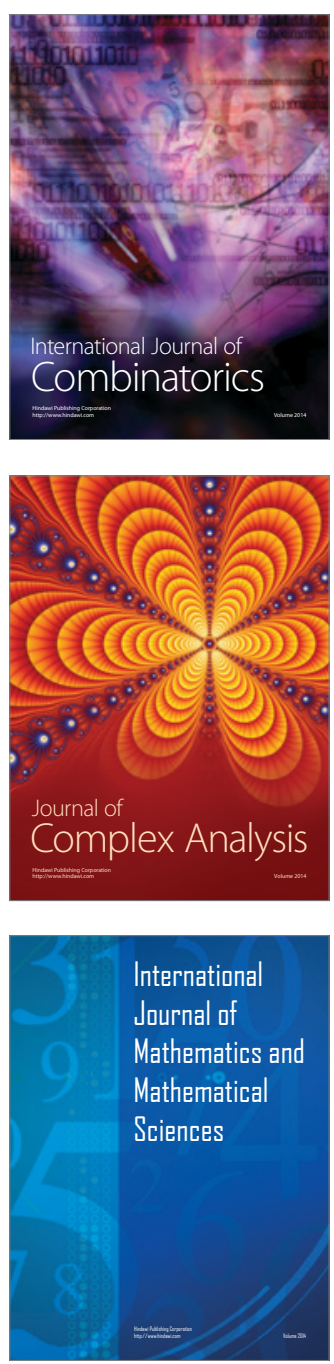
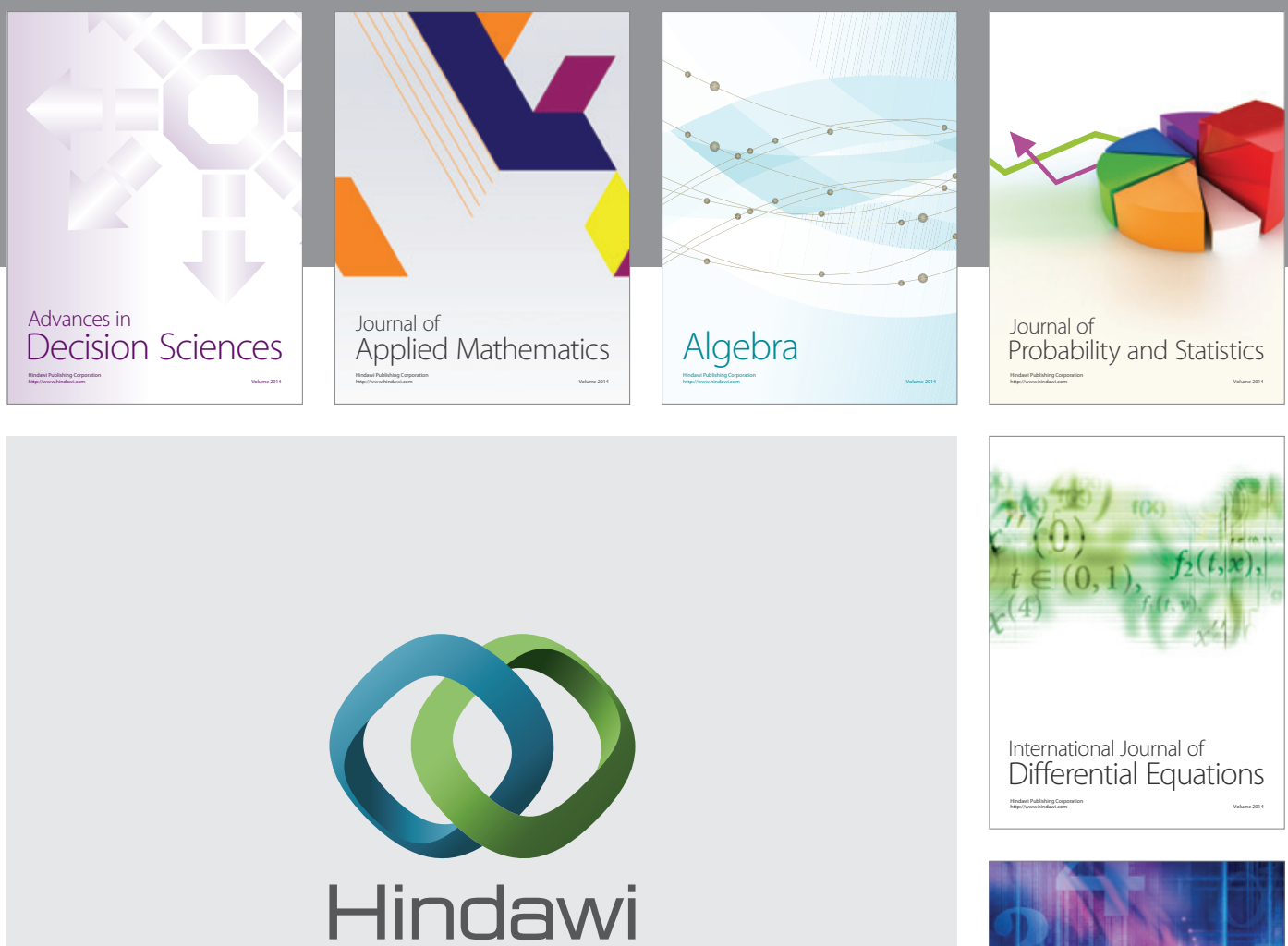

Submit your manuscripts at http://www.hindawi.com
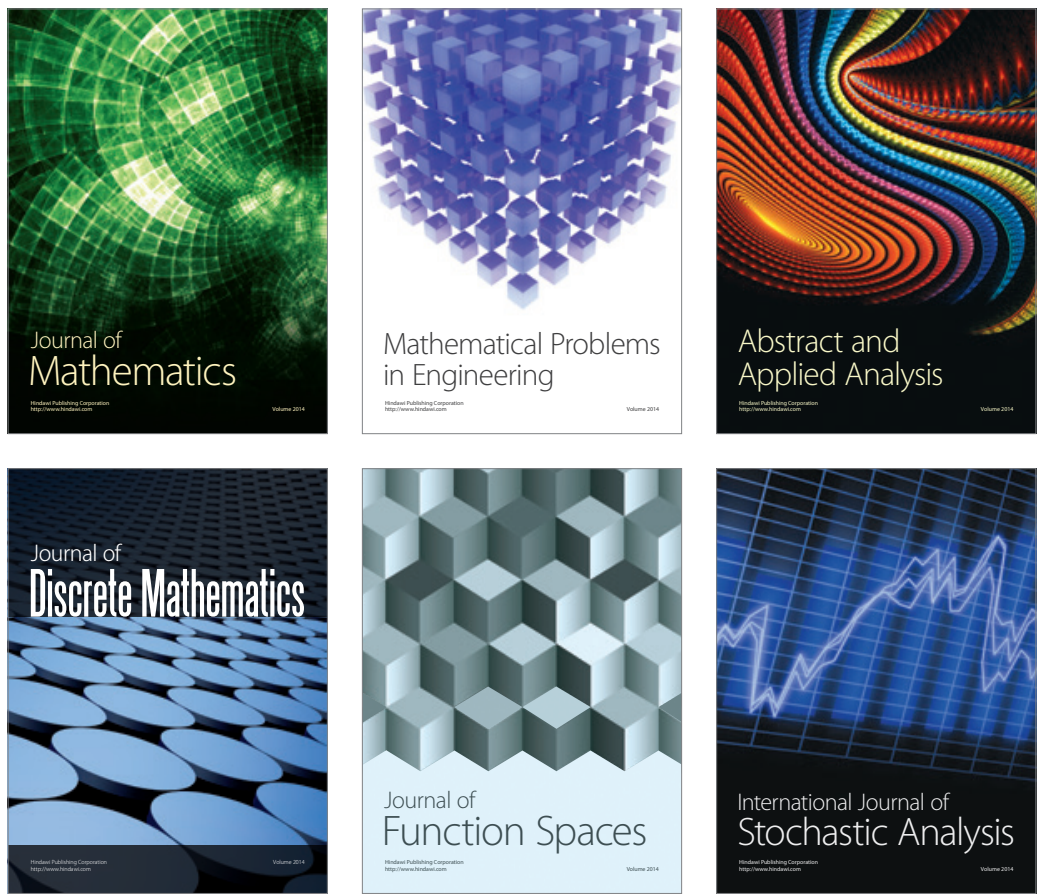

Journal of

Function Spaces

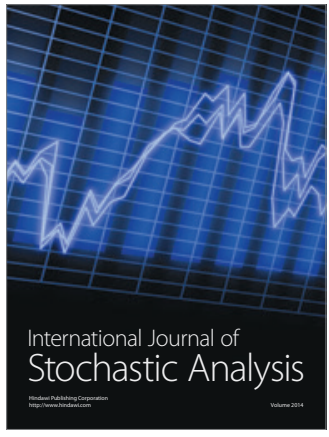

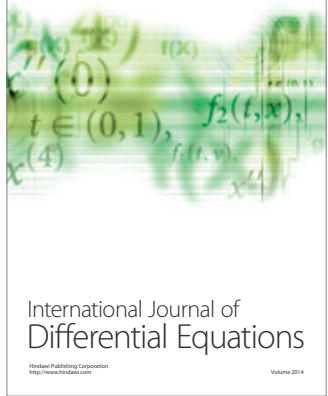
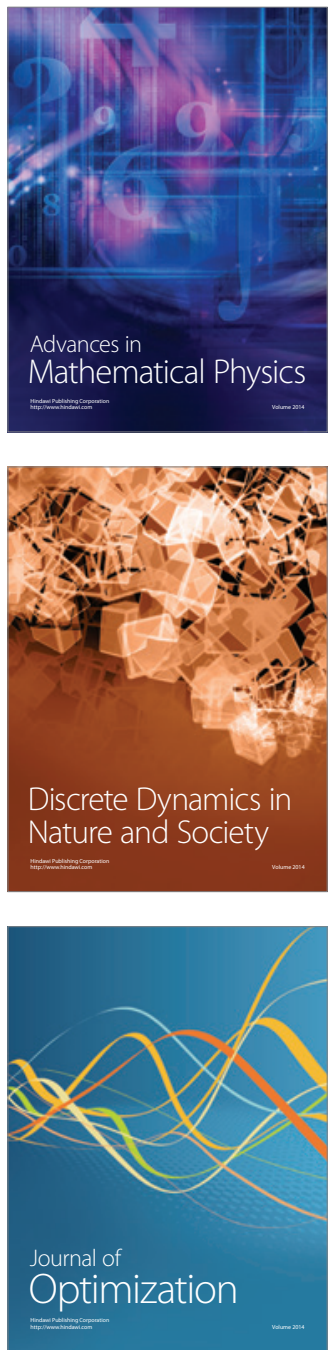